Check for updates

Cite this: RSC Adv., 2019, 9, 10528

\title{
Berberine nanoparticles for promising sonodynamic therapy of a HeLa xenograft tumour $†$
}

\author{
Hanqing Liu, ${ }^{a}$ Tingting Zheng, (DD *a Ziqian Zhou, ${ }^{a}$ Azhen $\mathrm{Hu}^{a}{ }^{a}$ Minghua $\mathrm{Li}^{a}{ }^{a}$ \\ Zhuxia Zhang, ${ }^{a}$ Guangyin Yu, ${ }^{b}$ Huanhuan Feng, ${ }^{c}$ Yawen An, ${ }^{d}$ Jiao Peng*e \\ and Yun Chen ${ }^{\star a}$
}

Here, we show that berberine (BBR) nanoparticles (BBRNPs, 300 nm hydrodynamic diameter) are a promising sonosensitizer for cancer sonodynamic therapy (SDT). HeLa cells were cultured for in vitro investigation, and a HeLa xenograft tumor model was established with BALB/c nude mice ( $20 \mathrm{~g}$, female) for in vivo study. Significant effects of BBRNP-mediated SDT were observed in both in vitro and in vivo experiments. Cell counting kit-8 (CCK-8) cell proliferation and cytotoxicity assays were performed to confirm if BBRNPs-SDT has cytotoxicity against HeLa cells in vitro. The mechanism for inhibition of tumor proliferation by BBRNPs-SDT was investigated via flow cytometry, photoluminescence spectroscopy, dynamic light scattering, scanning electron microscopy, ultrasonic contrast imaging, tumour pathological analysis, western blot and anatomical analysis. We identified two ongoing assumptive mechanisms. One is due to the tumor angioembolism effect, which blocks oxygen and nutrient supply in situ, leading to early-stage HeLa apoptosis. The other domino effect is due to ultrasonic energy-activated BBRNP cavitation and reactive oxygen species release, which leads to tumor vascular injury and finally induces HeLa apoptosis, resulting in tumour shrinkage. Both pathways synergistically helped with HeLa xenograft tumor supression. In conclusion, we posit that BBRNPs are a promising agent for tumor SDT.

Received 13th November 2018

Accepted 6th March 2019

DOI: 10.1039/c8ra09172b

rsc.li/rsc-advances cause tumor microvascular embolization (TME). Moreover, BBRNPs-SDT cancer treatment not only releases ${ }^{1} \mathrm{O}_{2}$ under focused ultrasound, but also triggers BBRNP cavitation and causes tumour vascular injury, leading to HeLa cell apoptosis and suppression of tumor proliferation.

In order to verify our concept, we studied berberine selfassembly both in aqueous media and in vivo, then checked BBR's effect on ROS through both photoluminescence and CCK8 experiments. During our research, the toxicity of berberine in vivo was tested in C57/B6 mice by analyzing the organ weight coefficient data. Next, the morphology study of BBR aggregation in aqueous media was performed via dynamic light scattering (DLS) and scanning electron microscopy (SEM). Further, HeLa cells were cultured for sonotoxicity estimation in vitro. After we confirmed that BBR indeed caused either cytotoxicity or biotoxicity in both the cell and mouse model, we introduced SDT for BBR combination therapy in xenografted HeLa mice. Finally, through BBR-SDT therapy, we discovered that the size of the xenograft tumor was significantly suppressed. On the other hand, a series of evidence, for instance, ultrasound angiography, cancer tissue H\&E staining and histopathological analysis all confirmed that BBRNPs first cause tumor angioembolism and subsequently activate cavitation and release ROS under SDT. As a result of BBRNPS-SDT treatment, HeLa cell morphology is significantly destroyed via both
China.E-mail: pengjiao153@163.com

$\dagger$ Electronic supplementary information (ESI) available. See DOI: 10.1039/c8ra09172b

${ }^{a}$ Shenzhen Key Laboratory for Drug Addiction and Medication Safety, Peking University Shenzhen Hospital, Shenzhen Peking University-Hong Kong University of Science and gmail.com; prof_yunchen@yeah.net

${ }^{b}$ Department of Pathology, Peking University Shenzhen Hospital, Shenzhen 518036, ${ }^{d}$ The State Key Laboratory of Chemical Oncogenomics, Key Laboratory of Genomics, School of Chemical Biology and Biotechnology, Peking University Shenzhen Graduate School, Shenzhen 518055, China

Shenzhen Graduate School, Shenzhen 518055, China 
mechanical damage from ultrasound as well as ROS membrane damage (see Scheme 1).

We found that berberine nanoparticles can accumulate progressively via the EPR (enhanced permeability and retention) effect and aggregate in tumor blood vessels due to the acidic microenvironment in the cancer tissue. ${ }^{16}$ Though it is reported that berberine can release ROS by photodynamic therapy, our research showed that both BBR cavitation and ROS can be driven by sonodynamic therapy in vivo. ${ }^{17,18}$ All the information we gained in this study indicates that BBRNPs-SDT might present a promising and better noninvasive tumor therapy approach for both preclinical and clinical cancer studies.

\section{Materials and methods}

\section{Reagents}

Berberine hydrochloride was purchased from Sigma-Aldrich Corporation (Burlington, MA, USA). Primary antibodies for western blot analysis were purchased from Invitrogen Corporation (Carlsbad, CA, USA). Peroxidase-conjugated goat antirabbit IgG was purchased from Jackson ImmunoResearch (West Grove, PA, USA).

\section{Mice}

C57/B6 mice (both male and female) and BALB/c nude mice (female) were provided by Guangdong Medical Laboratory Animal Center (Foshan city, Guangdong, China). Animals were allowed to acclimatize to the environment for 5 days before treatment with $a d$ libitum access to food and water. A $12 \mathrm{~h}$ light/dark cycle at 19.2$25.1{ }^{\circ} \mathrm{C}$ and a relative humidity of $42-59 \%$ were maintained. The study protocol was performed in accordance with the International Guidelines on the Care and Use of Animals and was approved by the Ethics Committee of Shenzhen PKU-HKUST Medical Center.

\section{Cell culture}

The human cervical cancer cell line, HeLa, was provided by The Hong Kong University of Science and Technology. HeLa cells were cultured in Dulbecco's modified Eagle's medium (Gibco, Carlsbad, CA, USA) supplemented with $10 \%$ fetal bovine serum (Gibco, Carlsbad, CA, USA).

\section{BBR self-assembly and morphology study}

BBR has irregular microstructures when it is dissolved naturally in aqueous media (see ESI Fig. S1 $\dagger$ ) due to its poor solubility in water. The defect leads to a uncontrollable injection dosage given during treatment. However, it has been reported that BBR can have regular nanostructures, known as BBR nanoparticles (BBRNPs), by triggering its self-assembly in neutral buffer. ${ }^{19}$ Sahibzada's group first reported that BBRNPs can be generated via both syringe pumping anti-solvent precipitation (APSP) and evaporative precipitation of nanosuspension (EPN).$^{20}$ Here we introduced an easier way to prepare BBRNPs. In order to prepare different concentrations of BBRNP stock solution, for instance $2 \mathrm{~mL} 100 \mu \mathrm{g} \mathrm{mL} \mathrm{g}^{-1}$ BBRNP stock, $200 \mu \mathrm{g}$ of BBR was weighed and mixed with $2 \mathrm{~mL}$ of neutral PBS buffer; the resulting turbid liquid was sonicated in a $55{ }^{\circ} \mathrm{C}$ water bath for 25 min until a transparent yellowish solution was generated. After cooling the BBR suspension to room temperature, dynamic light scattering (DLS) was performed to estimate the hydrodynamic diameter of the BBRNPs, and their nanoscopic morphological images were next produced by scanning electron microscopy (SEM). DLS measurements were estimated at $25^{\circ} \mathrm{C}$ using a Malvern Zetasizer Nano ZS Zen3500 equipped with a Peltier-controlled thermostatic cell holder. Parameters foe the DLS measurements were consistent with previous publications. ${ }^{21-25} \mathrm{~A} 633 \mathrm{~nm}$ laser and $173^{\circ}$ scattering angle were set as fixed parameters. For each measurement, the sample was allowed to have 2 min of equilibration time. Further, BBR nanoparticles were captured via SEM measurements. The SEM images were recorded on a FEI Magellan 400 field-emission SEM at an acceleration voltage of $5.0 \mathrm{kV} .{ }^{26}$ To prepare samples, we first rinsed and dried the assembled structures onto semiconductor adhesive tape for SEM. Samples were then sputter-coated with a $12 \mathrm{~nm}$ layer of iridium to avoid charging. ${ }^{22}$

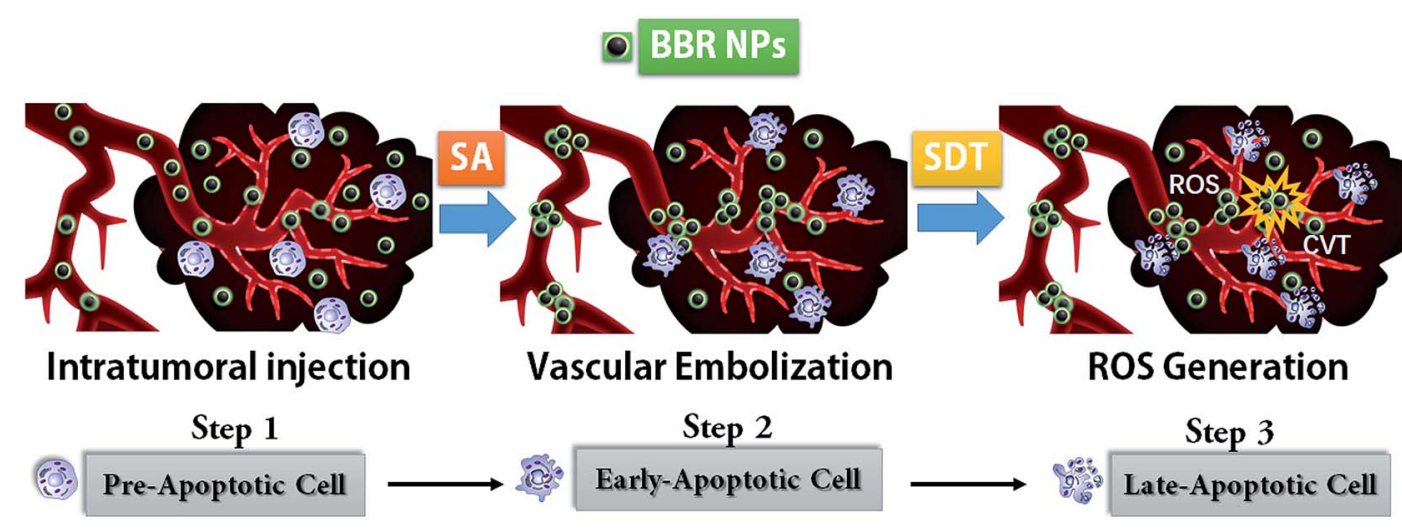

Scheme 1 Schematic diagram of how BBRNPs-SDT treatment works. (A) BBR oligomers enter tumour blood vessels via blood circulation. (B) Tumor vascular embolism forms according to BBRNP accumulation via an enhanced permeability and retention (EPR) pathway. Tumor angioembolism induces early HeLa apoptosis. (C) BBRNPs-SDT therapy leads to ROS release, which yields late HeLa apoptosis. BBRNPs stands for berberine nanoparticles, SA stands for self-assembly, SDT stands for sonodynamic therapy, ROS stands for reactive oxygen species, while CVT stands for cavitation. 


\section{Estimation of BBR sonotoxicity against HeLa}

HeLa cell proliferation and apoptosis under treatment with BBRNPs-SDT were evaluated. Cells $\left(1 \times 10^{6}\right.$ cells per $\left.\mathrm{mL}\right)$ were inoculated in 96-well culture plates for $24 \mathrm{~h}$, then berberine colloidal solutions at concentrations of $0,1.56,3.13,6.25,12.50$, 25.00, 50.00, and $100.00 \mu \mathrm{g} \mathrm{mL}{ }^{-1}$ were added. Cells were divided into BBR and SDT groups. After incubation for $6 \mathrm{~h}$, cells in the SDT group were exposed to ultrasound at a frequency of 1.0 $\mathrm{MHz}\left(1 \mathrm{~W} \mathrm{~cm}^{-2}\right)$ using an AFG3022B Ultrasonic Signal Generator (Tektronix Inc., Beaverton, OR, USA) for $60 \mathrm{~s}{ }^{27}$ and cells in the BBR group were not exposed to ultrasound, as a control. After $24 \mathrm{~h}$, a cell counting kit (CCK)-8 (DOJINDO, Tokyo, Japan) was used to measure the effects of the BBRNPs (hereafter, BBR) and BBRNPs-SDT (hereafter, SDT) on HeLa cell proliferation. A Spectra Max i3x microplate reader (Molecular Devices, Shanghai, China) was used to determine the absorbance at $450 \mathrm{~nm}$, and the reference wavelength was set at $405 \mathrm{~nm}$. Fluorescence spectroscopy (FLS980) was conducted to detect free oxygen radicals, and a laser channel with a $450 \mathrm{~nm}$ excitation wavelength was applied.

The PE Annexin V apoptosis detection kit I (BD Pharmingen $^{\mathrm{TM}}$, San Diego, CA, USA) was used to detect the presence of apoptotic cells. HeLa cells were cultured in 6-well plates and treated as above. Following $6 \mathrm{~h}$ of treatment, the cells were collected and resuspended in binding buffer. Then, $5 \mu \mathrm{L}$ of PE Annexin V and $5 \mu \mathrm{L}$ of 7-AAD per $1 \mathrm{~mL}$ were added successively. Cells were incubated in the dark at room temperature (RT) for $15 \mathrm{~min}$. Then, flow cytometry was performed to identify and select apoptotic cells.

Western blot analysis was used to test the effects of BBR and SDT on the protein expression phosphorylation levels of the signaling pathway. Briefly, HeLa cells were inoculated in 6-well plates ( $1 \mathrm{~mL}$ per well) for $24 \mathrm{~h}$. Cells in the BBR and SDT groups were treated with BBR of $0,1.56$, and $6.25 \mu \mathrm{g} \mathrm{mL}{ }^{-1}$. SDT treatment was performed as described above. The cells were collected and incubated at $4{ }^{\circ} \mathrm{C}$ for $30 \mathrm{~min}$ with lysis buffer (Thermo Fisher Scientific, Waltham, MA, USA) containing $1 \mathrm{mM}$ phenyl methane sulfonyl fluoride (Thermo Fisher Scientific, USA), $1 \mathrm{mM} \mathrm{Na}_{3} \mathrm{VO}_{4}$ (Sigma-Aldrich Corporation), and $20 \mathrm{mM}$ NaF (Sigma-Aldrich Corporation) 24 hours after treatment. The mixture was then centrifuged at $12000 \mathrm{rpm}$ for $30 \mathrm{~min}$. Protein concentrations were measured using a microspectrophotometer. $10.00 \mu \mathrm{L}$ aliquots of the samples were loaded into the wells of polyacrylamide gels and separated by electrophoresis at 80-120 V. Afterwards, the isolated proteins were transferred to membranes at a constant current of $330 \mathrm{~mA}$ for $90 \mathrm{~min}$, which were blocked for $1 \mathrm{~h}$ at RT, incubated with primary antibodies (overnight at $4{ }^{\circ} \mathrm{C}$ ) and then secondary antibodies ( $1 \mathrm{~h}$ at $\mathrm{RT}$ ), and detected using a chemiluminescence detector (Tanon 5220S; Guangzhou Ewell Bio-technology Co., Ltd., Guangzhou, Guangdong, China). The gray values of the protein bands were measured.

\section{BBR toxicology evaluation in vivo}

C57/B6 mice were given intraperitoneal (i.p.) injections with BBR in order to perform the toxicology evaluation. C57/B6 mice (half male and female) were divided into 7 groups according to the doses of BBR administration. BBR with doses of 0 (as control), 5, 10, 15, 20, 25, and $30 \mathrm{mg} \mathrm{kg}^{-1}$ were injected intraperitoneally, then the mice were observed for 7 days. The times and numbers of animal deaths in each group were recorded, and the median lethal dose $\left(\mathrm{LD}_{50}\right)$ was calculated. Deceased animals were dissected, and their hearts, livers, spleens, lungs, kidneys, stomachs, intestines, and genital systems were observed carefully. The heart, liver, spleen, lungs, and kidneys were dried and weighed to calculate the organ coefficients. The surviving animals were sacrificed after 7 days, and the aforementioned parameters were measured and observed.

\section{Antitumor effect evaluation in a xenograft mouse model}

In order to establish the xenograft tumor model, HeLa cells $(3 \times$ $10^{7}$ cells per $\mathrm{mL}$ ) were suspended in PBS containing $50 \%$ Matrigel (BD Biocoat ${ }^{\mathrm{TM}}$, San Diego, CA, USA) and subcutaneously implanted into 4 week-old BALB/c mice. Tumor growth was observed once a week after implantation, and tumor sizes were measured using a Vernier caliper. Treatments were performed when the tumor diameter reached about $1 \mathrm{~cm}$.

Mice with tumors were divided into 4 groups: control group (Ctrl), BBRNP group (BBR), ultrasound group (US), and BBRNPsSDT group (SDT). Mice in the BBR and SDT groups were injected with $10 \mathrm{mg} \mathrm{kg}^{-1}$ of BBR. After 24 hours, mice in the US and SDT groups were exposed to ultrasonic irradiation at a frequency of $1.00 \mathrm{MHz}\left(1 \mathrm{~W} \mathrm{~cm}^{-2}\right)$ for $60 \mathrm{~s}$.

The treatment effects on blood vessels were observed using a Vevo 2100 Imaging System (FUJIFILM Visual Sonics Inc., Toronto, ON, Canada). Microbubbles $(0.1 \mathrm{~mL})$ were injected intravenously, and the peak intensity and time to reach a peak in the tumor were measured to assess the degree of the opening of blood vessels. Two weeks after the second treatment, all of the animals were sacrificed and the tumors were removed and weighed to evaluate the therapeutic effects.

\section{Histological analysis}

The cancerous tissues were fixed in $4 \%$ paraformaldehyde immediately after removal from the nude mice. Then, they were dehydrated by successive incubation in $75 \%, 85 \%, 95 \%$, and $100 \%$ ethyl alcohol and xylene, followed by embedding in paraffin wax blocks. The tissue blocks were cut into $4 \mathrm{~mm}$-thick sections. Those sections were deparaffinized by xylene and rehydrated by successive incubation in 100\%, 95\%, and $75 \%$ ethyl alcohol. Hematoxylin-eosin (H\&E) staining of the cancer tissues was performed after the sections were deparaffinized and rehydrated, respectively. The images were observed under a light microscope (OLYMPUS, U-LHEAD, Japan) with $200 \times$ and/or $400 \times$ magnification.

\section{Statistical analysis}

Data are expressed as the means \pm standard deviation (SD), and one-way ANOVA analysis of variance was used to compare intergroup differences using SPSS 17.0 software (SPSS, Inc., Chicago, IL, USA). A probability ( $p$ ) value of $<0.05$ was considered statistically significant. 


\section{Results and discussion}

BBR is well-known as a natural dye with strong yellow fluorescence, as well as a traditional Chinese medicine that has been used since 3000 B.C. ${ }^{28}$ In recent decades, scientists have noticed that BBR can suppress tumor cell proliferation by releasing activated oxygen radicals, i.e. singlet oxygen ${ }^{1} \mathrm{O}_{2} \cdot{ }^{29,30} \mathrm{BBR}$ is commonly activated for ${ }^{1} \mathrm{O}_{2}$ release via optical excitation. Recently, scientists have found that ultrasound can activate berberine for release of reactive oxygen species (ROS) (Fig. 1A). ${ }^{\mathbf{1 5 , 3 1}}$ Nevertheless, anti-cancer reagents are always a double-edged sword and can be simultaneously harmful to normal cells. Therefore, we firstly performed BBR toxicology evaluation with C57/B6 mice and determined its safe dosage range for healthy organs. Then, we found that BBR significantly suppressed HeLa proliferation under the conditions of sonodynamic activation. Next, a HeLa xenografted nude mouse model was established for the investigation of BBR-SDT antitumour effects in vivo. The results show that SDT significantly suppresses tumor growth in xenografted mice. Mechanism studies showed that there might be two pathways contributing to this result. First, ultrasound contrast images showed that BBR nanoparticles authentically seal tumor microvessels right after SDT, which might temporarily block the nutrient supply of the surrounding tumor cells. Second, results from western blots showed that the PI3K-AKT-mTOR signaling pathway is triggered by SDT, resulting in the apoptosis of xenografted HeLa tumors in nude mice.

\section{BBRNP toxicological evaluation in vivo}

A BBR toxicity evaluation was performed first. Acute toxicity is an important parameter for drug use. Widespread organ damage was observed, especially in the heart, liver, spleen, lung, kidney, stomach and intestines (see ESI Fig. S2 $\dagger$ ). A large area of ischemia was seen in the liver of most of the animals and partly in the heart and kidney. Hemorrhagic spots could be seen in some gastrointestinal tracts. Pulmonary edema and enlarged spleens were observed in every dead animal and some surviving animals (Fig. 1).

Organ coefficients for the heart, liver, lung and kidney in every administration group (with BBR doses of 5, 10, 15, 20, 25, $30 \mathrm{mg} \mathrm{kg}^{-1}$ ) were significantly higher than those in the control group (Fig. 1); however, for the spleen coefficient, only the $30 \mathrm{mg} \mathrm{kg} \mathrm{kg}^{-1}$ administration of BBR exhibited a significant impact. No animals died when the BBR dose was $\leq 10 \mathrm{mg} \mathrm{kg}^{-1}$, and when the dose was $\geq 15 \mathrm{mg} \mathrm{kg}^{-1}$, animal deaths increased with increasing BBR doses. Meanwhile, the average time of death decreased. The vast majority of deaths occurred within 48 hours after administration. The $\mathrm{LD}_{50}$ of BBR administered via intraperitoneal injection was $17.012(15.122-18.700) \mathrm{mg} \mathrm{kg}^{-1}$ in mice. Therefore, a dose of $10 \mathrm{mg} \mathrm{kg}^{-1}$ was used to evaluate the antitumor effects of SDT in vivo.

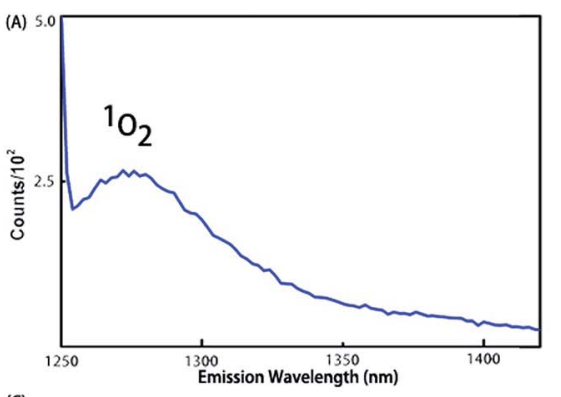

(C)

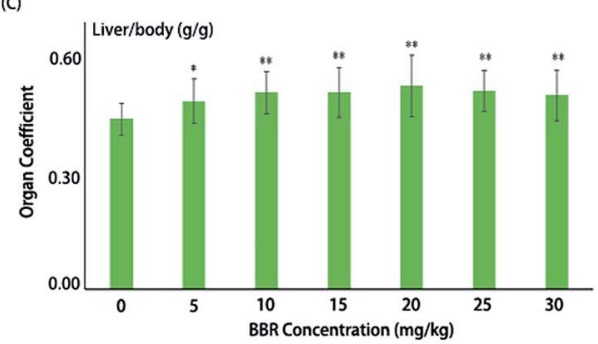

(E)

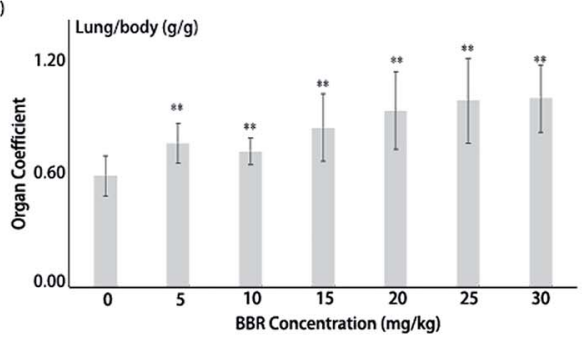

(B)
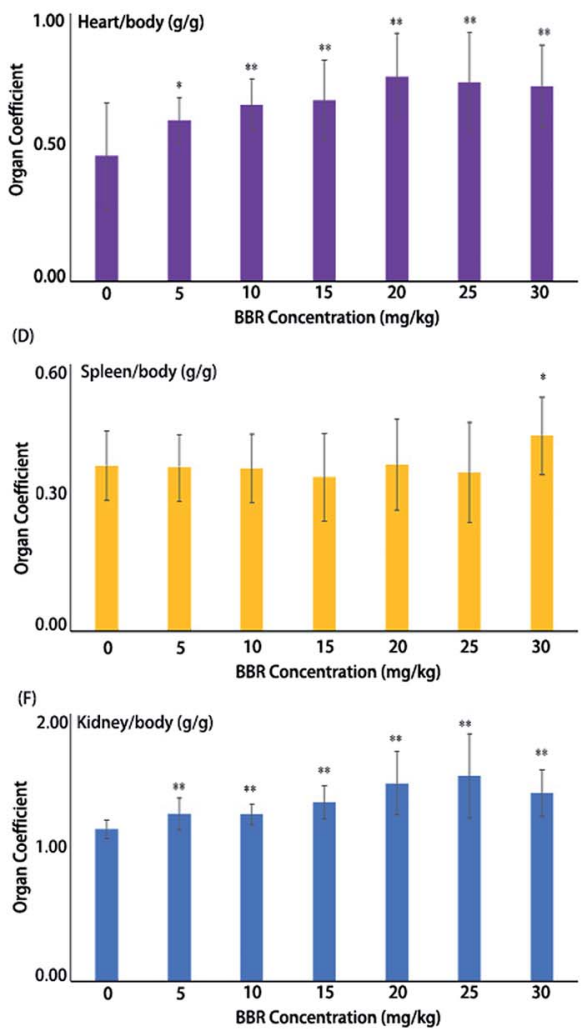

Fig. 1 In vivo toxicity of BBR (A) photoluminescence steady-state emission spectra of ${ }^{1} \mathrm{O}_{2}\left(\lambda_{\mathrm{em}}=1270 \mathrm{~nm}\right)$ generated from ultrasound-activated BBR. (B)-(F) Acute organ damage from BBR via intraperitoneal injection. Data for representative viscera and statistical results of organ coefficients are presented. ${ }^{*} P<0.05$ and $* * P<0.01 \mathrm{vs}$. control group. Organ coefficients indicate organ/body weight ratio ( $\mathrm{g} \mathrm{g}^{-1}$ ). 


\section{Antitumor effects of BBRNPs-SDT in HeLa cells both in vitro and in vivo}

Results of CCK-8 and flow cytometry (FCW) tests showed that both BBR and SDT inhibited cell proliferation and promoted cell apoptosis of HeLa cells in a concentration-dependent manner, and that the efficiencies of SDT treatments were higher than those of BBR at the same concentrations (Fig. 2A and $\mathrm{B})$. Furthermore, in vivo validation was performed in the xenograft model of HeLa. Tumor sizes and weights in the SDT group reduced significantly compared to those of the control group $(P<0.05)$. However, neither BBR without ultrasound nor ultrasound application only showed remarkable therapeutic effects $(P>0.05$, Fig. 2C and D). These results indicated that ultrasound could promote the inhibiting effects of BBR in HeLa.

\section{Effects of BBRNPS-SDT on tumor blood vessels}

The ultrasound imaging system was used to observe the peak intensity and time to peak of microbubbles in the tumor after intravenous injection, which reflects the blood flow and degree of opening of tumor blood vessels. As shown in Fig. 2, neither BBR nor ultrasound alone significantly changed the amounts of microbubbles entering the HeLa tumor, but microbubbles in the tumor of SDT group significantly decreased compared with the control group (Fig. 2B), the contrast mean power after microbubble injections of SDT group was also significantly lower than that of the control group (Fig. 2D). Both BBR and SDT seemed to partly reduce the time to the peak of the microbubbles, but there was no statistically significant differences (Fig. 2C). These results suggested that SDT may have the effect of sealing tumor blood vessels, thus reducing the nutrient supply in the tumor and starving the tumor cells.

H\&E staining was further performed to show the significant changes in vascular obstruction with the SDT groups. The control group is shown in Fig. 3(E-1), in which the cancer cells can be seen to have regular morphology, indicating that the cancer tissue is growing healthily. However, vessel thrombosis (Fig. 3(E-2), yellow arrow) is present in the BBR treatment group, where HeLa cells around the blocked vessel are in the early stage of apoptosis (Fig. 3(E-2), white frame). Besides this, significant coagulation necrosis and calcification (Fig. 3(E-3), yellow arrows) appeared when we introduced focused ultrasound for treatment, and the HeLa cytomembranes in the focal area were also damaged, as shown in the white frame of (E-3). Nevertheless, none of these situations led to thorough HeLa destruction, only when we introduced both BBR and focused ultrasound together did this occur (Fig. 3(E-4)). HeLa cells underwent significant apoptosis during BBR-SDT treatment, not only due to FUS (focused ultrasound) mechanical damage, which leads to cancer tissue devastation, but also due to BBR ROS cytotoxicity, yielding both ultrasound and ROStargeted cancer SDT therapy.

Fig. 3(E-2) shows significant tumor vascular obstruction, while Fig. 3(E-3) and (E-4) also show extensive necrotic areas, in addition to the clots. Large numbers of red blood cells were observed in the necrotic area, which was probably due to ultrasound destruction of microcirculation and perfusion. The vascular structure was not damaged, and no thrombus was seen
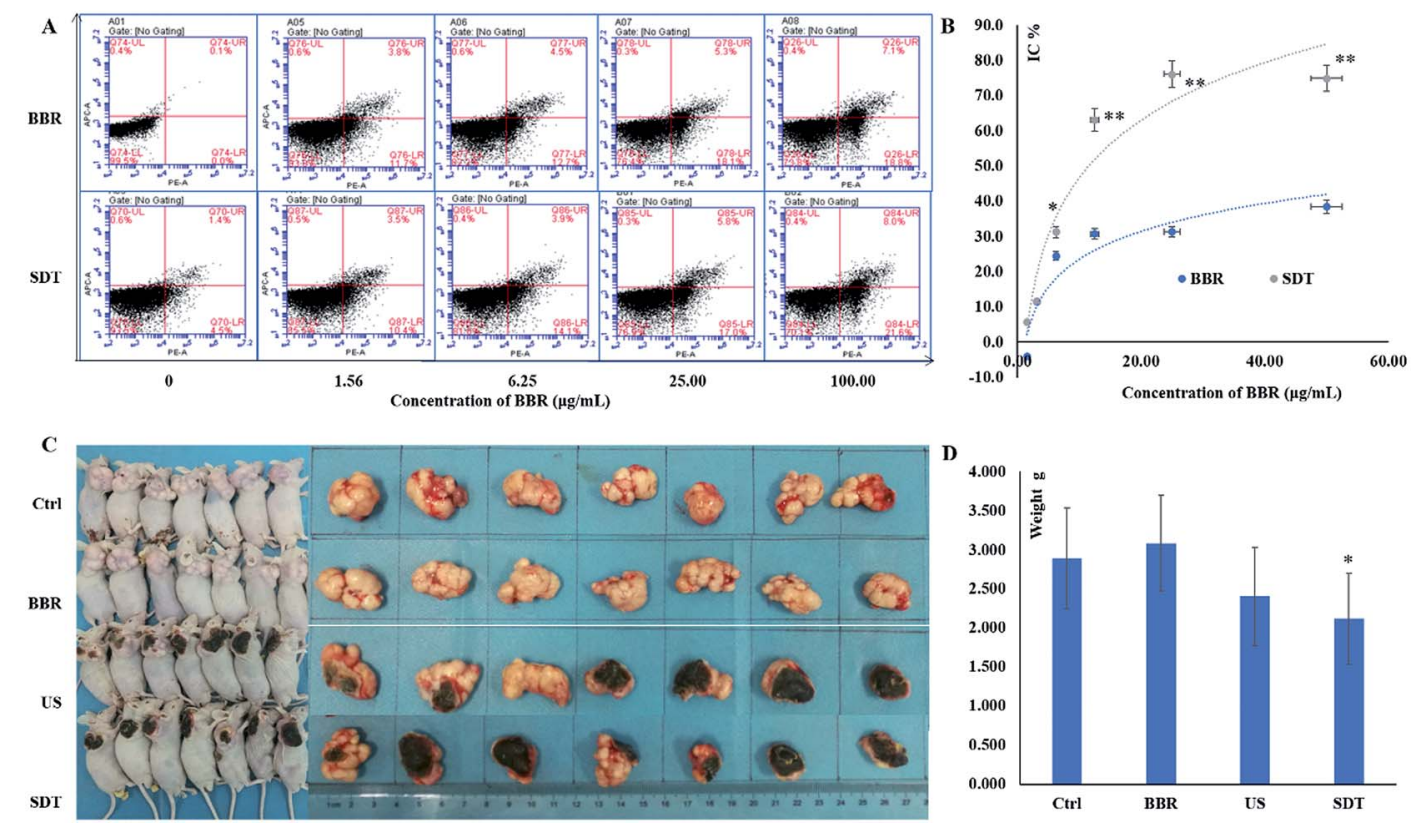

Fig. 2 Antitumor effects of BBR-SDT in HeLa. (A) Apoptosis test; concentrations of BBR were 0, 1.56, 6.25, 25.00 and 100.00 $\mu \mathrm{g} \mathrm{mL}{ }^{-1}$; the PE Annexin V positive cell populations were considered as the apoptotic cells. (B) Cell proliferation inhibition; concentrations of BBR were 0, 1.56, $3.13,6.25,12.50,25.00,50.00$ and $100.00 \mu \mathrm{g} \mathrm{mL}^{-1}$; CCK- 8 assay was used, and absorbance was detected at $450 \mathrm{~nm}$ with reference wavelength of $405 \mathrm{~nm}, * P<0.05$ and $* * P<0.01$, vs. BBR group. (C) Effects on xenograft model; dose of BBR was $10 \mathrm{mg} \mathrm{kg}^{-1}$; ultrasonic irradiation parameters: frequency of $1.00 \mathrm{MHz}\left(1 \mathrm{~W} \mathrm{~cm}^{-2}\right)$ for $60 \mathrm{~s}$. (D) Comparison of tumor weights in different groups, $N=7, \mathrm{means} \pm \mathrm{SD}, * P<0.05, v s$. control group. Ctrl: control group, BBR: BBR group, US: ultrasound group, SDT: BBR-mediated SDT group. (PE: R-phycoerythrin, APC: allophycocyanin) H\&E stained histopathological section charts of burned tissue are shown in ESI, Fig. S5. $\dagger$ 
(A)

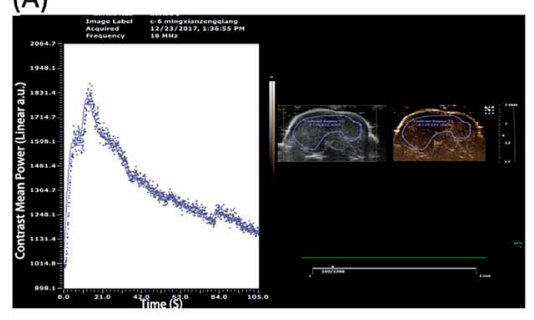

(D-1)

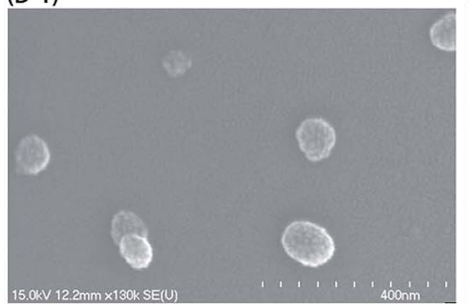

(E-1)

(E-2)
(B)

(D-2)
(B)
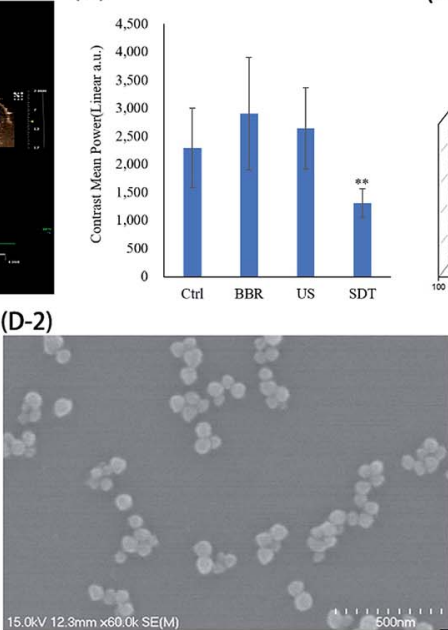

(E-3)

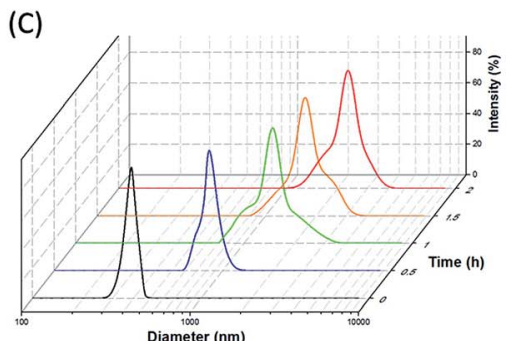

(D-3)

Diameter $(\mathrm{nm})$

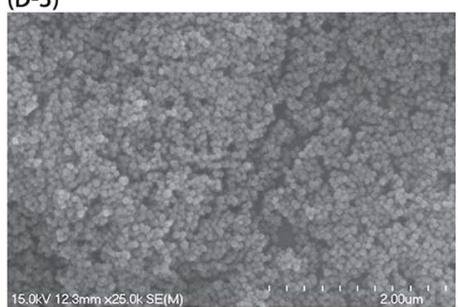

(E-4)

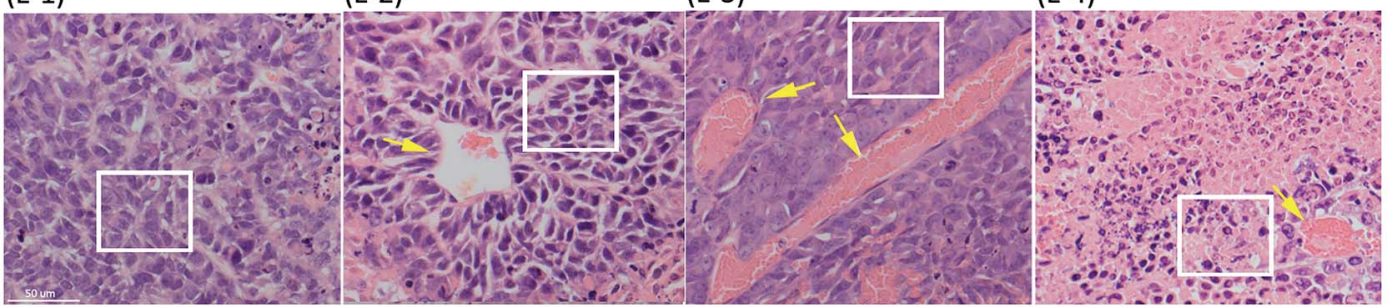

Fig. 3 Analysis of tumor vascular morphology changes due to BBRNPs-SDT treatment. (A) Representative images of ultrasound examination and microbubble imaging; the contrast mean power and time to peak after microbubbles injections were recorded; the injection volumes of microbubbles were $0.1 \mathrm{~mL}$. (B) Comparison of microbubble imaging in different groups; acquisition time of imaging was $5 \mathrm{~s}$ after microbubble injection. (C) BBR hydrodynamic diameter ( $\mathrm{nm}$ ) increases with time. (D) SEM photography of BBR monomers and poly aggregates with increasing time. (E) E-1 to E-4 present H\&E staining histological analysis of cancer tissue with control, BBR, US and BBR-US treatment, respectively. Yellow arrows point to mutant blood vessels; white bar indicates $50 \mu \mathrm{m}$, while white frame highlights the HeLa cells. See ESI (Fig. S5†) for pathological sections of burned cancer tissue and BBRNP cavitation-damaged cancer tissue.

in the other groups. Our results fully demonstrated both vascular obstruction and tumor necrosis in the SDT groups.

One of the potential mechanisms of BBRNPs involved in antitumor effects in vivo might relate to blocking the blood vessels in the tumor. ${ }^{32-34}$ The effects of ultrasound on blood vessels have been well known, mainly involving sonoporation and thermal effects. Ultrasound imaging in our study suggested that SDT led to blood vessel closure, limiting tumor growth by reducing the nutritional supply of the tumor. On the other hand, vascular closure may lead to a decrease in the oxygen concentration in the tumor tissues, which is not conducive to the production of ROS and the killing effects of SDT on tumor cells. Therefore, the selection of appropriate BBR and ultrasonic parameters between the closure and opening of blood vessels may be one of the directions worth further exploring.

\section{Effects of BBRNPs-SDT on signaling pathways}

The results of in vitro and in vivo studies confirmed that ultrasound could enhance the antitumor effects of BBR. To reveal the potential mechanisms for the effects of BBR-SDT on the signaling pathways of cell proliferation and apoptosis, protein expression and phosphorylation levels of phosphoinositol 3 kinase (PI3K), protein kinase B (AKT), the mammalian target of rapamycin compounds 1 (mTORC1), p70 ribosomal S6 kinase (p70s6K), ribosome protein S6 (rps6), 4E binding protein 1 (4EBP1) and protein synthesis initiation factor 4E (eIF4E) were analyzed using western blot analysis. As shown in Fig. 4, cascade phosphorylation of PI3K, AKT, mTORC1, p70s6K, rps6, 4EBP1 and eIF4E were inhibited by BBR, and ultrasound amplified this inhibition effect. This indicates that the signaling pathway PI3K-AKT-mTOR, which has important regulatory effects in both cell proliferation and apoptosis, was inhibited by BBR and BBR-SDT.

The PI3K-AKT-mTOR signaling pathway is widely known to play a key role in translation and cell proliferation. In fact, there have been many studies on the effects of BBR on this signaling pathway. For example, $\mathrm{Yu}^{35}$ found that the BBR-induced apoptotic and autophagic death of HepG2 cells requires inhibition of mTORC1 via AMP-activated protein kinase (AMPK) activation. Shukla ${ }^{36}$ observed that BBR induced downmodulation of the PI3K-AKT-mTOR pathway of HepG2 cells. Chitra $^{37}$ revealed that BBR increased Beclin-1 and LC3-II levels and enhanced autophagosome formation, indicating that autophagy stimulation is attributed to inhibition of p-mTOR. The results of Yi' $\mathrm{s}^{38}$ study showed that BBR inhibited the AKTmTOR-p70s6k-rps6 pathway and activated the mitochondrial apoptosis pathway, which might explain the antitumor effect of 

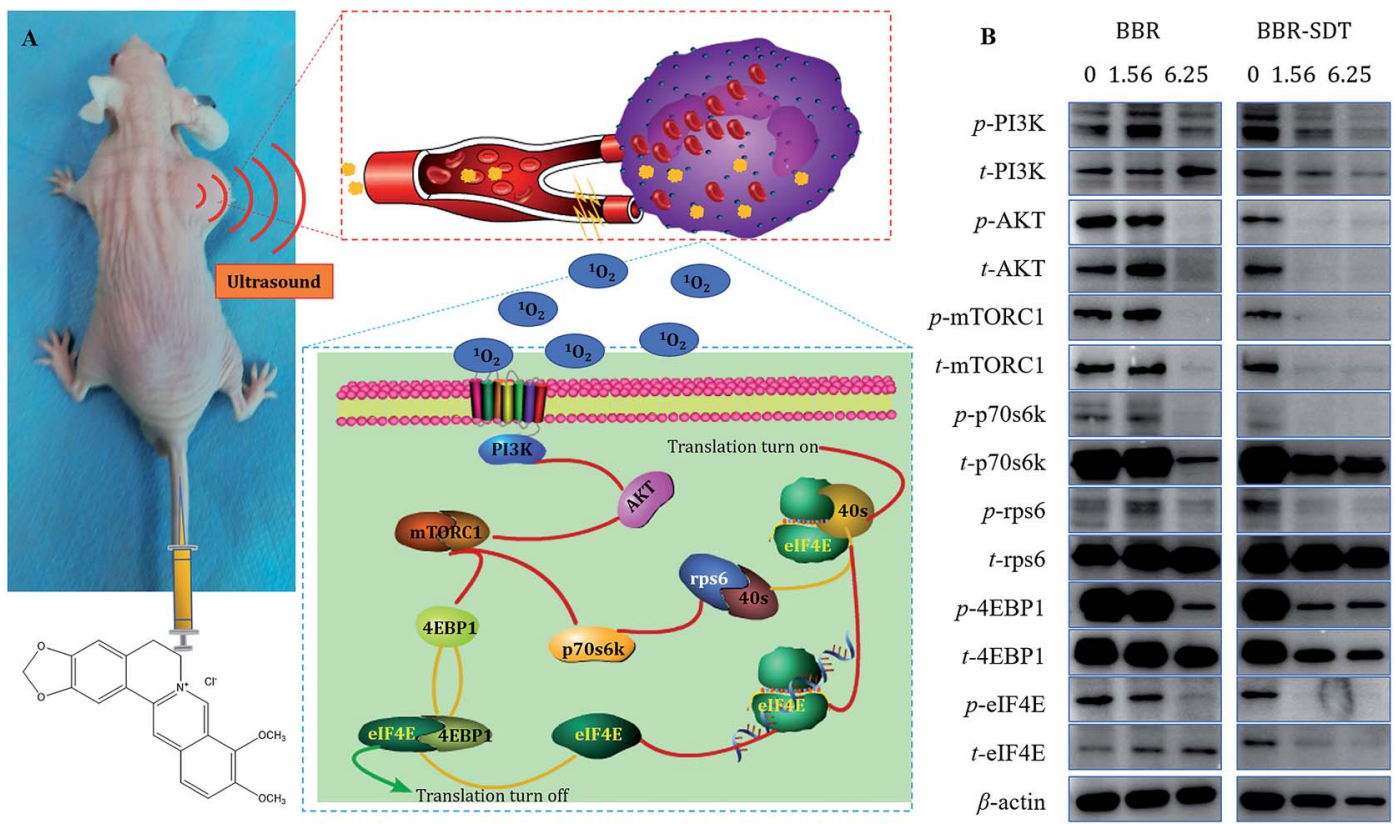

Fig. 4 Inhibition of the PI3K-AKT-mTOR signaling pathway by BBR-SDT. (A) Diagrammatic representation. (B) Western blot analysis. Concentrations of BBR used were $0,1.56$, and $6.25 \mu \mathrm{g} \mathrm{mL}^{-1}$, and coincubation treatments were performed for $6 \mathrm{~h}$.

BBR on human gastric cancer cells. Chen $^{39}$ found that BBR inhibited proliferation and induced apoptosis of U20S cells in a dose-dependent manner by inhibiting the activation of the PI3K-AKT pathway and regulating the expression of Bax, Bcl-2 and caspase 3. These studies support our findings that BBR inhibited the phosphorylation of the PI3K-AKT-mTOR signaling pathway and the downstream molecules in a dose-dependent manner. Besides this, inhibition by SDT of PI3K-AKT-mTOR phosphorylation was more obvious than that by BBR. This pathway might be one of the potential mechanisms for the inhibition of cell proliferation by SDT. ${ }^{40}$

\section{Conclusions}

We observed the acute toxicity of BBR administered via intraperitoneal injection and defined the safe and effective injection dose of BBR. Our study may provide some references for its future application.

We confirmed that BBR is a promising sonosensitizer which mediates SDT, showing antitumor effects both in vitro and in vivo, and its potential mechanisms might be related to inhibiting PI3K-AKT-mTOR signaling pathways and blocking tumor blood vessels.

\section{Conflicts of interest}

There are no conflicts to declare.

\section{Acknowledgements}

We acknowledge financial support from the following grants: the Natural Science Foundation of China No. 81871358, the China Postdoc Fund No. 2018M640807, the
Science \& Technology Key Project No. 2016YFC0104707, the Guangdong Bureau of Traditional Chinese Medicine Project No. 20171228, the Natural Science Foundation of Guangdong No. 2018A0303130228, the Guangdong Medical Research Project No. B2018090, the Shenzhen Science and Technology Planning Project No. JCYJ2018022318126494, No. JCYJ20180507183224565, No. JCYJ20160427185055877, No. JCYJ20160429090753103, No. JCYJ20170413100222613, the Health and Family Planning Commission of Shenzhen No. 201605018, and the Shenzhen "Sanming" Project No. SZSM201512026.

\section{References}

1 T. Suzuki, S. Kamada, Y. Yoshida and K. Unno, Heterocycles, 1994, 38, 1209-1211.

2 D. Costley, C. Mc Ewan, C. Fowley, A. P. McHale, J. Atchison, N. Nomikou and J. F. Callan, Int. J. Hyperthermia, 2015, 31, 107-117.

3 A. P. McHale, J. F. Callan, N. Nomikou, C. Fowley and B. Callan, Adv. Exp. Med. Biol., 2016, 880, 429-450.

4 L. Rengeng, Z. Qianyu, L. Yuehong, P. Zhongzhong and L. Libo, Photodiagn. Photodyn. Ther., 2017, 19, 159-166.

5 H. Shibaguchi, H. Tsuru and M. Kuroki, Anticancer Res., 2011, 31, 2425-2429.

6 M. Trendowski, Cancer Metastasis Rev., 2014, 33, 143-160.

7 G. Canavese, A. Ancona, L. Racca, M. Canta, B. Dumontel, F. Barbaresco, T. Limongi and V. Cauda, Chem. Eng. J., 2018, 340, 155-172.

8 Y. A. Larasati, N. Yoneda-Kato, I. Nakamae, T. Yokoyama, E. Meiyanto and J.-y. Kato, Sci. Rep., 2018, 8, 2039.

9 C. Geng, Y. Zhang, T. H. Hidru, L. Zhi, M. Tao, L. Zou, C. Chen, H. Li and Y. Liu, Life Sci., 2018, 207, 304-313. 
10 J. Qian and Q. Gao, Ultrasound Med. Biol., 2018, 44, 853-860. 11 L. Dai, Y. Wei, C. Sun, L. Mao, D. J. McClements and Y. Gao, Food Hydrocolloids, 2018, 85, 75-85.

12 S. Hajigholami, Z. V. Malekshahi, N. Bodaghabadi, F. Najafi, H. Shirzad and M. Sadeghizadeh, Iran. J. Pharm. Res., 2018, 17, 1-10.

13 X. Li, L. Gao, L. Zheng, J. Kou, X. Zhu, Y. Jiang, Z. Zhong, J. Dan, H. Xu, Y. Yang, H. Li, S. Shi, W. Cao, Y. Zhao, Y. Tian and L. Yang, Int. J. Nanomed., 2015, 10, 821-838.

14 X. Li, X. Zhang, L. Zheng, J. Kou, Z. Zhong, Y. Jiang, W. Wang, Z. Dong, Z. Liu, X. Han, J. Li, Y. Tian, Y. Zhao and L. Yang, Cell Death Dis., 2016, 7, 1-12.

15 J. Y. Kou, Y. Li, Z. Y. Zhong, Y. Q. Jiang, X. S. Li, X. B. Han, Z. N. Liu, Y. Tian and L. M. Yang, Cell Death Dis., 2017, 8, e2558.

16 H. Lee, A. F. Shields, B. A. Siegel, K. D. Miller, I. Krop, C. X. Ma, P. M. Lorusso, P. N. Munster, K. Campbell and D. F. Gaddy, Clin. Cancer Res., 2017, 23(15), 4190-4202.

17 N. L. Andreazza, C. Vevert-Bizet, G. Bourg-Heckly, F. Sureau, M. J. Salvador and S. Bonneau, Int. J. Pharm., 2016, 510, 240249.

18 R. Bhattacharyya, B. Saha, M. Tyagi, S. K. Bandyopadhyay, B. S. Patro and S. Chattopadhyay, Free Radical Res., 2017, 51, 723-738.

19 M. U. K. Sahibzada, A. Sadiq, H. S. Faidah, M. Khurram, M. U. Amin, A. Haseeb and M. Kakar, Drug Des., Dev. Ther., 2018, 12, 303-312.

20 M. U. K. Sahibzada, A. Sadiq, H. S. Faidah, M. Khurram, M. U. Amin, A. Haseeb and M. Kakar, Drug Des., Dev. Ther., 2018, 12, 303-312.

21 T. Zheng, F. P. P. Martinez, I. M. Storm, W. Rombouts, J. Sprakel, R. Schirhagl and R. D. Vries, Anal. Chem., 2017, 89(23), 12812-12820.

22 T. Zheng, H. Feng, J. M. V. D. Broek, K. Rahimi, A. J. C. Kuehne, R. D. Vries and J. Sprakel, Macromol. Rapid Commun., 2018, 1800284.

23 T. Zheng, J. Voskuhl, F. Versluis, H. R. Zope, I. Tomatsu, H. R. Marsden and A. Kros, Chem. Commun., 2013, 49, 3649.

24 T. Zheng, A. Boyle, M. H. Robson, D. Valdink, G. Martelli, J. Raap and A. Kros, Org. Biomol. Chem., 2015, 13, 1159-1168.
25 T. Zheng, M. Bulacu, G. Daudey, F. Versluis, J. Voskuhl, G. Martelli, J. Raap, G. J. A. Sevink, A. Kros and A. L. Boyle, RSC Adv., 2016, 6, 7990-7998.

26 Y. Wang, C. Zhou, W. Wang, D. D. Xu, F. Y. Zeng, C. Zhan, J. H. Gu, M. Y. Li, W. W. Zhao, J. H. Zhang, J. H. Guo, H. H. Feng and X. Ma, Angew. Chem., Int. Ed., 2018, 57, 13110-13113.

27 H. Chen, X. Zhou, Y. Gao, B. Zheng, F. Tang and J. Huang, Drug Discovery Today, 2014, 19, 502-509.

28 C. Rajagopal, M. B. Lankadasari, J. M. Aranjani and K. B. Harikumar, Pharmacol. Res., 2018, 130, 273-291.

29 J. Jiao, D. Xu, Y. Liu, W. Zhao, J. Zhang, T. Zheng, H. Feng and X. Ma, Micromachines, 2018, 9, 83.

30 S. Saxena, S. Shukla and P. Kakkar, Toxicol. Appl. Pharmacol., 2018, 347, 92-103.

31 X. Li, L. Yang, Y. Tian, X. Zhu, L. Zheng and J. Kou, Nanoparticles containing berberine release system useful for preparing sound sensitizer in sonodynamic therapy for atherosclerosis; and in preparing carrier, CN10553535974A, Univ. Harbin Medical, 2016.

32 H. Zhang, K. Liu, Z. Xue, H. Yin, H. Dong, W. Jin, X. Shi and H. Wang, Am. J. Transl. Res., 2018, 10, 334-351.

33 B. Gu, W. Wu, G. Xu, G. Feng, F. Yin, P. H. J. Chong, J. Qu, K. T. Yong and B. Liu, Adv. Mater., 2017, 29, 26.

34 Q. Zou, H. Zhao, Y. Zhao, Y. Fang, D. Chen, J. Ren, X. Wang, Y. Wang, Y. Gu and F. Wu, J. Med. Chem., 2015, 58, 79497958.

35 R. Yu, Z. Q. Zhang, B. Wang, H. X. Jiang, L. Cheng and L. M. Shen, Cancer Cell Int., 2014, 14, 1475-2867.

36 S. Shukla, F. Rizvi, S. Raisuddin and P. Kakkar, Free Radical Biol. Med., 2014, 76, 185-199.

37 P. Chitra, G. Saiprasad, R. Manikandan and G. Sudhandiran, J. Mol. Med., 2015, 93, 1015-1031.

38 T. Yi, L. Zhuang, G. Song, B. Zhang, G. Li and T. Hu, Nutr. Cancer, 2015, 67, 523-531.

39 Z. Z. Chen, Iran. J. Public Health, 2016, 45, 578-585.

40 H. Chen, X. Zhou, Y. Gao, B. Zheng, F. Tang and J. Huang, Drug Discovery Today, 2014, 19, 502-509. 